Voix et Images

volxetimages

\title{
La poésie de Monique Bosco : une quête de justice
}

\section{Joseph Bonenfant}

Volume 9, numéro 3, printemps 1984

Monique Bosco

URI : https://id.erudit.org/iderudit/200476ar

DOI : https://doi.org/10.7202/200476ar

Aller au sommaire du numéro

Éditeur(s)

Université du Québec à Montréal

ISSN

0318-9201 (imprimé)

1705-933X (numérique)

Découvrir la revue

Citer cet article

Bonenfant, J. (1984). La poésie de Monique Bosco : une quête de justice. Voix et Images, 9(3), 13-21. https://doi.org/10.7202/200476ar d'utilisation que vous pouvez consulter en ligne.

https://apropos.erudit.org/fr/usagers/politique-dutilisation/ 


\title{
La poésie de Monique Bosco: une quête de justice
}

\author{
par Joseph Bonenfant, Université de Sherbrooke
}

À l'orée de l'oeuvre poétique de Monique Bosco, on se demande si on ne pourra jamais en tirer la même substance que de l'oeuvre romanesque. On a raison, mais la question se pose autrement. Disons que la même substance pourrait être le malheur humain. C'est une hypothèse. On retrouvera ce malheur et dans les romans et dans les poèmes, mais ces derniers le traduiront du plus vif du langage, tandis que les romans le moduleront à travers des personnages en situations. Les changements de forme auront tellement modifié cette substance qu'ils seront eux-mêmes devenus ce qui la remplace: des champs langagiers inassimilables.

Etablissons aussi que la poésie de Bosco n'a pas obtenu, à ce jour, la reconnaissance qu'elle mérite. Schabbat parle moins haut qu'Un amour maladroit; Jéricho n'éveille pas le même écho que Charles Lévy, m.d., que New Medea, ou que la Femme de Loth. Assurément. Les poèmes publiés dans des revues n'existent peut-être pas. Il y a un défi à relever. On peut toujours avancer que cette poésie a davantage proposé son étrangeté que sa familiarité. Une limpidité et une retenue, une timidité et une sincérité dont l'institution n'a su que faire. Possible. On ne pourrait le savoir en toute certitude que si on dressait un tableau complet des best-sellers poétiques des vingt dernières années. Peut-être qu'une lueur d'explication viendrait au jour.

Mieux vaut sans doute, pour résoudre ces difficultés que je semble soulever à loisir en guise de préliminaires, adhérer franchement à un énoncé critique de René Char, affirmant: «Avec Rimbaud, la poésie a cessé d'être un genre littéraire, une compétition». Ainsi la question de l'importance relative de la poésie de Bosco se trouve-t-elle résolue, et par rapport à la réception critique dont elle a joui, ou non, et par rapport à l'ensemble de l'oeuvre romanesque dont elle se démarque de façon autonome tel un con- 
trepoint musical. Le roman allonge ce que la poésie condense, dilue dans de multiples destins ce qu'elle concentre dans un seul, et s'appuie sur des perspectives sociologiques qu'elle ne considère pas, pour elle-même, prioritaires. De plus, je ne connais pas, chez Bosco, de discours sur la poésie, comme on peut en trouver chez Anne Hébert, Paul-Marie Lapointe, Roland Giguère ou Nicole Brossard. On est à pied d'oeuvre devant un monument qui dissimule ses accès. Autant se résoudre à ce défaut d'accès pour se forcer à une adhésion plus obstinée. Char affirme encore: «Nous obéissons librement au pouvoir des poèmes et nous les aimons par force».

\section{La souffrance juive. La souffrance femme}

Les dix-huit poèmes parus dans Écrits du Canada français en 1963 (no 15, pp. 169-190) modulent à leur manière, rapide et directe, les confidences malheureuses déjà faites dans le premier roman, Un amour maladroit (Gallimard; 1961). C'est la révélation d'une vie de ruptures et d'arrachement, un chemin d'errances et d'étapes comme seul en a connu, et connaît toujours, le destin juif. Mais ici une femme parle. Le premier poème, «l'amour des temps premiers», signale la perte «de l'amour entrevu au seuil d'une aube tranquille». On dirait la fin d'un monde, l'évanouissement du bonheur. Aux strophes de ce premier poème de révélation succèdent les paragraphes du deuxième texte, non titré, qu'on pourrait dire d'explication, et s'ouvrent dans la plus grande transparence de l'énonciation. «Je suis fille de la terre où rien ne pousse. Fille stérile des sables du désert». Voici peutêtre le texte le plus lyrique de Monique Bosco, étant donné l'interpellation du coeur («t'en souvient-il, mon coeur?»; "ai-je jamais eu une âme, mon coeur?», etc.). Le discours tire sa virulence des relations $j e-t u$, non inter, mais intra-personnelles. «Pas d'enfance pour moi, ni de jeux ni de rires». Et tant de larmes imbibant la terre. "Alors vraiment, nous étions le sel de la terre». Ironie amère vraiment propre à la souffrance juive.

Le destin individuel se fond dans le destin collectif de 1'Israèl des siècles. «Souviens-toi des lueurs de crématoire. Des cendres à n'en plus finir». À ce destin de mort s'ajoute le destin de vie. «De si longues faims étirées au cours des siècles. Et la soif au désert. Et le froid tout au long des âges». Au passage est démasquée l'injustice, déploré l'exil en terre étrangère, dénoncée la fraude et le jeu truqué, dont est victime un peuple et une femme de ce peuple. Le dernier poème de cette suite, également sans titre, propose une autre position transparente de la subjectivité; mais cette fois avec la disparition totale du je, et une espèce de recourbement du lyrisme sur lui-même qui arrive à une froide résolution. À la première décision qui impliquait l'énonciatrice dans son discours: "Il faudrait devenir sourde et muette. Statue de sel au pays du mensonge», succède une série de voeux sapientiaux, descriptifs, résolutoires, d'une tendresse émouvante: 
«Sage sagesse, aux gestes sévères, aux consignes immuables... Douce et seule sagesse de l'indifférence et de la répétition». Et la poète, rappelant les "pièges d'ombre et de souffrance" et la "source unique au goût du malheur", voue le malheur en même temps que la sagesse:

Voilà la seule liqueur amère et quotidienne capable d'étancher une certaine soif d'enfance, de vengeance, d'oubli et de pardon pour laquelle la seule sagesse est le silence.

Il y a de la grandeur à assumer ainsi son destin. Dans cet acte de sagesse, que ne renierait ni un Qohéleth, ni un Jérémie, ni un Raymond Abellio, et tant d'autres, se profile l'acte poétique, issu du silence et y ramenant toujours, l'acte le plus total n'étant possible que par la parole, à l'égal d'une foi, ou d'un amour. La souffrance demeure, ni escamotée ni rachetée, élément premier de l'aventure humaine, tant collective qu'individuelle, ici accrue par le fait capital de la féminitude. La souffrance juive. La souffrance femme.

Entre ces deux textes posés comme des bornes éclairantes autant à ce premier recueil qu'à l'ensemble de l'oeuvre, se donnent à lire quinze poèmes, titrés, brefs, rythmés, sortes de chants d'accompagnement issus d'un travail de plaisir, en dépit de leur contenu. Quinze poèmes qui sont autant d'énoncés de vérité et de certitude, avec des titres impératifs: «Laide est la ville. En terre étrangère, Règne du silence, L'amour condamné, Fantôme d'enfance, Asiles, Écartèlement, Coeur obscur», etc., titres qui thématisent le discours poétique en vertu d'un travail de l'auteure facilitant la lecture, mais qui aussi manifestent autant la "dispersion du sujet» que «sa continuité avec lui-même», pour reprendre les expressions de Michel Foucault. Le seul travail prosodique, dans ces poèmes intercalaires, empêche qu'on ne voie dans ce discours que l'expression d'un sujet. Autrement dit, le poème est linguistiquement, et sémiotiquement, autonome; il existe, une fois terminé, hors du feu nourricier, ou de l'eau-mère, de l'énonciation. Que le je d'un poème se dise «à l'étroit en (sa) peau», ou qu'il s'oppose «au règne du silence», qu'il crie à travers la ville, ou déclare la perte des «jeux innocents, pervers/Années perdues, enfouies», ou affirme: «je crie en vain les horreurs que je vois», ou assume le destin femme:

Je suivrai l'exemple triste

De celles qui m'ont précédée

Étrange communauté de filles

Liées ensemble par la défaite

Et l'attente des lendemains,

que le je, donc, multiplie ses occurrences et se déploie dans toutes ses facettes, ici prioritairement déclaratives, il ne fonde pas le discours comme expression du sujet, ni comme son enfermement. Au contraire, le discours ap- 
parait, selon l'heureuse formule de Michel Foucault, comme «un champ de régularités pour diverses positions de subjectivité». C'est ce qui rend possible la lecture, en d'autres mots l'intérêt d'un énonciataire, vous et moi, finalement l'histoire.

Je ne vois pas comment cette suite poétique de 1963 aurait pu recevoir l'accueil «critique» qu'elle méritait, ni comment elle aurait pu s'intégrer harmonieusement dans le champ littéraire québécois, étant donné la violence idéologique qui marquait le passage de la poésie québécoise du stade nationaliste au stade révolutionnaire (1963 est l'année de fondation de Parti pris), étant donné aussi le silence historique des femmes. Comment la judaîté errante aurait-elle pu enfoncer son coin dans la québécité sédentaire? Le malheur québécois, planant pratiquement au-dessus de l'histoire, pouvait-il s'accroître du malheur juif, ancré dans l'histoire planétaire? S'ajoutant à cette carence, au Québec comme ailleurs, on peut mentionner l'oppression d'un système patriarcal qui occultait, déformait ou raturait la parole femme. Bosco n'avait en principe aucune chance de son côté. C'est la patience de l'écrivaine, romancière et poète, qui à la longue vainc l'apathie générale. Des temps plus réceptifs sont venus qui donnent maintenant accès à cette oeuvre.

\section{Le refus du bonheur}

Il faut accorder de l'attention aux onze poèmes parus dans la revue Europe en 1969 (février-mars, pp. 185-190, consacrée à la littérature du Québec). De nouveau on remarque les titres fortement thématiques. La majorité des poèmes exposent des phrases à la syntaxe ample et précise, recourant à de significatives et suggestives symétries:

\section{À tous, tous les torts. Un barbelé nous défend du monde des vivants. \\ À nous, les remords. Conscience en bandoulière, poursuivons la tournée des morts.}

De nouveau, le malheur courtise les frontières. «Fini le temps de la vie. Fini le temps du repos. (...) Fini le temps de la joie». Les pertes vont s'accumulant: la joie du jeu, le coeur, la patrie, la possession, la famille, le soleil, les enfances: tout cela envolé. Le tableau n'est cependant pas parfaitement noir. Il faut noter deux éclaircies, comme des aubes inespérées. «Jardins de juin» présente l'énonciatrice, «douce et sage, délires domptés, peurs dénouées", "comme un jardin qui se meurt, au matin, pour renaître victorieux à la lune». "Nul pèlerinage» la présente, uniquement par la marque du féminin à deux participes passés, avec un coeur «noyé, dans le lac lisse et 
glauque de l'oubli ancien, peureux et poreux". Mais surprise, comme une interruption de la durée: "Voila que la vie fleurit en un miracle d'amour et d'oubli»». Ces résurrections, pour ambiguës qu'elles soient, méritent considération en vertu de leur rareté.

On n'exagérerait certes pas en affirmant que la poésie de Bosco marque un obstiné refus du bonheur. Cette position volontairement consentie n'est pas étrangère à la poésie, étant donné la distance réelle qui sépare l'énoncé de l'énonciation. Le premier n'est pas le reflet, ni même l'envers, de la seconde. Quand je lis: «Je chanterai les mensonges de l'amour,/moi qui ne chante guère», ou encore: «J'erre dans les déserts du vide de mes enfances», je lis des énoncés de Monique Bosco, mais proposés dans l'instance poétique. Il ne faut pas que les dimensions confidentielles des romans obstruent les avenues du sens poétique, ni que les éléments du poème soient vus comme fragments autobiographiques. De tels énoncés, certes, souffrent d'un excès référentiel, mais pas au point où ils ne puissent me rejoindre dans mon vécu existentiellement et historiquement autre, sexuellement différent, émotivement étranger. La judaîté, la féminité aident à lire un énoncé comme: «la route est libre. Les déserts de la patrie absente nous tendent les bras»; mais cela aussi s'entend en gentilité, en masculinité. Autrement dit, le sens se forme à l'écart du réel; ou plutôt le réel fait trace sur le sens, pas davantage, si lourdement ou avec insistance que ce soit. La poésie est un acte de liberté.

\title{
Les saisons et les éléments du réel
}

Jéricho, le premier livre-recueil de poèmes de Bosco, paraît en 1971, chez HMH. Quarante-six textes, des proses poétiques pour la plupart, quelques rares poèmes identifiables visuellement. La sagesse vouée antérieurement est à portée d'atteinte, empreinte d'une sérénité omniprésente. (Je me souviens de la ferveur de ma première lecture). L'auteure n'a pas cru bon de dégager des parties, de proposer des équilibres. Le plaisir d'écrire se manifeste par les moyens d'une économie syntaxique sans faiblesse. Il est temps de dire que la technique littéraire de Bosco doit tout à la syntaxe:

\begin{abstract}
Rêves d'aurore. Heureux présages. La peur s'estompe. Tel un mirage miroite le lac tranquille d'une oasis de calme. À l'horizon, un ciel sans nuage. Ce matin, il faisait gris et noir. Le grand soleil du midi a effacé les traces du malheur («Comme un mirage», p. 61).
\end{abstract}

Règne de la parataxe, mais jamais avec les effets d'essoufflement que donnent certains poèmes de Saint-Denys Garneau. Presque jamais de marqueurs jonctifs (et, car) ou disjonctifs (mais, cependant). Les circonstants de lieu et de temps sont utilisés sans excès. Cette coupe syntaxique coïncide avec un régime métaphorique minimal. Rarement peut-on lire une poésie 
avec si peu d'enflure, d'images sauvages. Des textes émondés, des énoncés épris de justesse, aspirant à l'heureux équilibre du dire et du dit, de l'impensé et du formulé, ennemis de l'ostentation. La poésie de Bosco exige une attention aux aguets; elle ne laisse rien au hasard et à la facilité. Poèmes de taille, phrases de taille, comme des pierres faisant de leur poids légèreté, et de leur masse un envol. Impression que le secret des formes propres a été percé, que les textes ont trouvé leur respiration profonde.

Réussite formelle qui n'est certes pas étrangère à l'élargissement et à l'approfondissement des contenus. On retrouve de nouveau les sentiments de l'usure et de l'irréparable, autres motifs de la perte, poétiquement acquise. Le poème liminaire, «Reprises», ouvre, même lexicalement, l'horizon féminin, corporel, des générations. «Inlassablement, il faut filer, tisser d'autres fils quand la trame se fait plus mince, transparente». Le temps compte plus que jamais auparavant, bien que constitué de jours ordinaires, d'échecs amoureux. Le passé a laissé des traces vives: «Le coeur battant encore. Au seul bruit de ton nom, il s'emballe tel un cheval fou de peur». La vie donne lieu à l'autodépréciation: «Je me noie dans ces infâmes cuisines où l'on fait réduire à petit feu les idiotes comme moi»; donne lieu, également, au désir de repos, fait à noter, consigné dans le dernier poème du recueil, «La paix des braves», et même dans ses dernières lignes: «Dans la trêve de la guerre à l'amour, je sais enfin que j'ai gagné la paix des braves n'attendant plus ni victoire ni défaite». «Paix des braves: paix honorable pour ceux qui se sont battus courageusement» (Petit Robert). Pas la mort, mais l'armistice immédiat, définitif. Pas la paix des cimetières, celle des jardins fleuris.

Cette quête de paix, fortement signalée en fin de recueil, on peut en suivre les traces à travers les poèmes. Il s'opère, dans ce recueil de Bosco, un retournement qu'on pourrait expliciter par l'opposition suivante: l'humanité (sinon la masculinité) ennemie versus la nature amie, cette dernière remplaçant la première, comme un bien remplace un mal, ou l'adoucit tout au moins.

Cette métamorphose met en scène le sentiment saisonnier. Le recours à la saison figure une sorte de confiance naturelle dans le retour de la vie et même dans sa requalification. On observe d'abord un niveau simple de métaphore, par le truchement du complément déterminatif: «aux champs en friche de l'amour, j'ai mené paître la fragile apparence du plaisir»; «aux marais salants de l'enfance, $j$ 'ai pourchassé le sanglant rêve d'autrefois»». Parfois une détermination plus condensée renforce la figure naturelle, comme les "pluies froides de la mort», ou «les grandes marées du désespoir». En de plus fréquentes occasions, c'est tout l'être intime qui trouve appui, recours et secours, force et pureté dans un élément naturel. «La liberté se cache derrière chaque buisson»; «l'aube seule voit la débâcle désordonnée des ombres transfuges de la mémoire»; «que tombe la première neige pour assourdir l'écho des paroles meurtrières»; «le grand soleil de midi a effacé 
les traces du malheur»; "des soleils hors de saison se rient des calendriers humains»; «lavée de pluie et de mer, je ris des peurs d'hier». La saison offre des climats, comme la nature des remparts, ou des baumes, toutes figures de métamorphoses et de résurrections.

Il en va de même pour la saisie du coeur utas de plumes", et du corps «tas de ferraille»; ou de la tête, «bille vide au duvet fou»; ou du «tintamarre des os entrechoqués». Dislocation, perte du lieu propre, mais jamais irrémédiable. Il semblerait qu'on puisse prêter à la vie, au corps, au coeur, ce que l'auteure prédique de la terre: «La terre se fend, s'étire, peau de chagrin prête aux plus folles métamorphoses». Si on met en parallèle, ou en opposition, ce rude espoir de vie avec certains textes aussi mortuaires que l'hiver:

Une fois de plus, la vie sera impossible, gâchée, bafouée. Vie vide de toute vertu. Vienne la mort. Vive son effarant silence. Il est temps de saluer avec reconnaissance l'avènement de l'absence,

on ne pourra savoir quelle saison, quel sentiment l'emporte. Est-ce la vie? Est-ce la mort? Le printemps («Le démenti des crocus paraît téméraire») a-t-il un règne imaginaire plus fort que l'hiver («Le pays s'éternise dans sa carapace d'hivern)? Il semble que non. Mais la vie éclate et s'acharne. Un élément en tout cas est constant, c'est l'eau purificatrice: "Que chaque vague lave la souillure de nos plus affreuses et intimes blessures» (p. 19), à quoi correspond, en fin de recueil, l'eau fortifiante, vivificatrice: «Trombes d'eau noyant le paysage. Rafales de vent rabattant loin et fort toute faiblesse offerte. Je retrouve une force enracinée en mon coeur enfin libre» (p. 62).

Jéricho met en scène une figuration ambivalente qui témoigne de la complexité du réel autant que de la poésie. Un univers univoque serait irrespirable. Il se tisse ainsi entre les êtres et les objets des contradictions fécondes, entre l'instant et le mouvement une dialectique vivante, entre les images du monde et celles de l'être intime une figuration écartelée, plurivoque. La poésie de Monique Bosco tire son pouvoir du scandale de l'esprit devant le réel autant que devant l'histoire. On ne peut, sur ces surfaces lisses, que laisser une empreinte fugace, une trace hâtive. La poésie fait délirer le tout autre du réel, l'esprit humain. Monique Bosco orchestre ce délire d'une main assurée, sans surcharge. 
La révolte contre une injustice immémoriale

Le dernier recueil paru aux Quinze, en 1978, Schabbat, 70-77, affiche, comme le précédent, une judaïté indéracinable. Dans Jéricho, le poème-titre (p. 45), annonce le renoncement au passé et à l'héritage. Mieux vaut le «pauvre présent» plutôt qu'une ville morte ou que «l'absurde futur incohérent». Dans Schabbat, c'est autant la femme que la juive qui «crie à l'injustice», et dénonce très fort son exclusion du temple: «Et je t'invoque, Seigneur, toi qui n'a pas cru bon de me faire place dans ton temple (...). Pas de place, dans ton temple, pour la femmequi a peiné, toute la semaine, dans le sang et la sueur. Le sabbat des hommes est sacré" (p. 94). Voilà une parole de révolte, ironique, sarcastique, ici condensée sur une phrase, mais éparse dans tout le recueil, le plus féroce, le plus autonome, le plus libérant, le plus construit de l'oeuvre poétique de Bosco.

Quatre parties. "Voyages d'exil» propose, avec «Venises», le rendez-vous raté de l'amour, la "dérisoire attente», le "corridor de l'angoisse", d'indifférence: «J'ai beau entendre tonner. Invincible à la foudre et au foutre, je nargue les éclairs du désir incertain»; avec «Florides», c'est le paysage maritime arpenté par les vieillards, «rescapés de la mort»; avec «Afriques», le voyage le plus beau, nous lisons des «choses vues», des êtres émouvants; c'est plein de couleurs, de saveurs et de chaleurs, bêtes mêlées aux humains. «Dans le marché noir de monde, les petits tas de tomates s'écrapoutissent sous le double assaut de la chaleur et des mouches». La deuxième partie, «Durs voyages aux pays de l'homme», redonne à lire le coeur saisonnier, ramène les vieillards, les paysages frappés ( Arbres noirs, droits, durs, drus et nus. Paysage de noir»), et encore, les pièges de l'amour. C'est la troisième partie, "Voyages aux bords de la mort», ${ }^{1}$ qu'on retiendra comme le cri de la femme blessée, pourfendeuse de l'injustice immémoriale, le cri solidaire, prophétique, contre l'assujettissement des femmes, leur asservissement, leur oppression, leur exclusion. À travers la chaîne, et les chaînes, des générations: "Amère petite juive, dressée à encaisser les coups. (...) $\mathrm{O}$ ma mère, dis-moi comment répondre. Tu nous as si scrupuleusement élevées que nous disons encore merci, de surcroît». Dans le Livre des Juges, c'est Dalila qui séduit, trahit, livre Samson. Dans le beau poème «Samson", la poète refuse "cette trop juste trahison", car elle a choisi Samson comme amant; elle le sait invincible, autant que le Samson historique qui, une fois rendu aveugle, ébranla les deux colonnes de l'édifice et fit mourir plus de trois mille hommes et femmes. «Une nuit de colère, c'est Samson qui me réglera sordidement mon compte. Je le sais depuis le commencement des temps». Cette science immémoriale se change en une conscience écorchée de l'injustice. Alors monte une plainte virulente, dans la «mise à mort», l'urgence d'un cri primal, foudroyant:

1. Les titres des $2 e$ et $3 e$ parties sont manifestement corrects aux pp. 43 et 67 ; ils ne le sont pas dans la table des matières, où il faut les intervertir. 
Et je commence tout juste à crier. Et vous n'avez pas fini de m'entendre. Je percerai vos tympans. Comme ce cri, trop longtemps retenu, vibrera sans merci à l'aube. Je crierai jusqu'à ce qu'on m'entende. (...) Rien n'entravera ma plainte. Je ne cherche ni vengeance ni pardon (p. 87).

Ces «voyages aux bords de la mort» forment, avec la quatrième et dernière partie, un tout unifié par la conscience militante et dénonciatrice des femmes et centré sur l'histoire de leur exclusion dans le système patriarcal. Monique Bosco a écrit, sous le titre "Odyssée des filles folles» (et le soustitre de dérision «semaine sainte») le plus construit, le plus virulent réquisitoire contre le mâle dominateur, dans la perspective biblique qu'elle connaît à fond, le plus assoiffé de justice, d'égalité. Dieu lui-mème, comme dans $J o b$, est mis en accusation, au premier chef: «Dieu exigeant. Impitoyable et avare. Ta parole est dure. Tout t'es da». Toute l'humanité femme est ici présentée comme victime; toute l'humanité juive aussi. «Longues nuits de Pologne et d'Allemagne. (...) Le peuple à la nuque raide courbe l'échine sous le double poids des lois humaines et divines». La guerre des sexes s'étend à la guerre opposant le ciel et la terre, à celle aussi qui divise les femmes: «Les saintes femmes se voilent la face devant le spectacle obscène de leurs soeurs possédées du démon». Le poème se termine abruptement sur une assertion généralisante qui a le mérite de signifier à sa manière un avertissement, une connaissance, une certitude, et une libération: "Tout homme, en cette heure du déclin du soleil, se transforme en Judas» (p. 98).

Cette dénonciation du mal universel et historique, ici le mal mâle, a le mérite, entre autres, d'ouvrir la conscience individuelle sur de larges horizons. Elle est prophétique, en ce sens qu'elle est faite au nom de toutes les femmes de toutes les générations humaines. Elle relève d'un savoir qui fait acte dans le réel, que dis-je, d'un savoir, d'un vouloir et d'un pouvoir tout neufs, promis à des réalisations, à des actualisations qu'on imagine encore mal.

À la fin de Schabbat, la phrase ci-haut citée convient, tel un point d'orgue qu'on peut prolonger à volonté, au besoin. Un paragraphe de la Femme de Loth (Laffont, 1970), autrefois, eat apporté, non une réconciliation, mais une nuance, une lueur d'arc-en-ciel. «Dieu t'a peut-être créé à son image. Pas moi. Je suis contente d'être femme, juive, sans pays, patrie, famille, biens. Sans rien. Trahie. Il ne me reste rien. Et je voudrais ouvrir les mains, vider mon coeur de toute rancoeur. Et te dire: «Va, je ne te hais point ${ }^{2} »$.

Est-il permis, est-il souhaitable, sans trahir la poète, de souscrire de tout coeur au voeu de la romancière?

2. Gloria Escomel cite aussi de texte dans le substantiel article qu'elle a consacré à Monique Bosco dans la Nouvelle Barre du jour, no 65, avril 1978, pp. 90-97. 